\title{
Mehrsprachige Schreib- und Text(sorten)kompetenz in curricularen Vorgaben, Sprachprüfungen, Sprachlehrwerken und im Sprachunterricht Teilergebnisse einer Vorstudie
}

\section{Věra Janíková}

The development of multilingualism has been considered a desirable and generally accepted goal, since it was declared a priority of language education policy by the Council of Europe and the European Union several years ago. This paper presents a qualitative empirical study which examines how the development of multilingual writing skills and the text competence manifests itself in strategic and curricular documents and standardized tests using the example of the text type Invitation; the extent to which this text type is addressed in language textbooks and trained in common classes of (foreign) languages. This investigation is located in the context of research on third language acquisition in the area of multilingual writing skills, and it is focused on the currently typical foreign language constellation in Czech language education, i.e. English as L2 and German as L3. The description of the methodological design of the study and the presentation of its preliminary results are preceded by a brief statement on the current concept of writing skills, writing competence and text competence as well as the multiple text and text type competence.

Writing skills - multilingual writing and text competence - multiple text (type) competence - research on third language acquisition - content analysis - text linguistics - curriculum language test - textbook

Die Entwicklung der Mehrsprachigkeit gilt als wünschenswertes und gesellschaftlich allgemein anerkanntes Ziel, seit sie vom Europarat und von der Europäischen Union schon vor mehreren Jahren zu einer sprachen- und bildungspolitischen Priorität erhoben wurde. In diesem Beitrag wird eine qualitative empirische Untersuchung vorgestellt, die zeigen soll, wie sich die Vermittlung bzw. Entwicklung der mehrsprachigen Schreib- und Textkompetenz am Bespiel der Textsorte schriftliche Einladung in bildungsstrategischen und curricularen Dokumenten sowie standardisierten Prüfungen manifestiert, inwieweit sie in Sprachlehrwerken aufgegriffen und im Rahmen des üblichen (Fremd)-Sprachenunterrichts trainiert wird. Die Untersuchung ist im Kontext der Tertiärsprachenforschung im Bereich des mehrsprachigen Schreibens zu verorten und konzentriert sich auf die zurzeit typische Fremdsprachenkonstellation in der tschechischen Sprachbildung, d. h. Englisch als L2 und Deutsch als L3. Der kurzen Beschreibung des Untersuchungsdesigns und der Präsentation der Teilergebnisse geht ein kurzes Statement voraus, wie die Schreibfertigkeit, die Schreibund Textkompetenz sowie die multiple Text(sorten)kompetenz in der aktuellen Sprachlehrund Sprachlernforschung betrachtet werden.

Schreibfertigkeit - mehrsprachige Schreib- und Textkompetenz - mehrsprachige Text(sorten) kompetenz - Tertiärsprachenforschung - Inhaltsanalyse - Textlinguistik - Curriculum Sprachprüfung - Lehrwerk 


\section{Zur Schreib- und Textkompetenz im Fremdsprachenunterricht}

Seitdem sich die kommunikative Didaktik im Fremdsprachenunterricht durchgesetzt hat, sind die vier sprachlichen Fertigkeiten Hören, Lesen, Sprechen und Schreiben „Fundament und ,tragendes" Moment des Fremdsprachenunterrichts" (Faistauer 2010: 960). Was die Fertigkeit Schreiben angeht, die in diesem Artikel im Zentrum des Interesses steht, wurde diese noch in den 70er Jahren des vorigen Jahrhunderts - in den Anfängen des Kommunikativen Ansatzes - vernachlässigt, u. a. auch deswegen, weil die gesprochene Sprache und eher mündliches Sprachhandeln im Vordergrund standen. Später, in der postkommunikativen Ära (Kumaravadivelu 2005; Funk 2010), wurden die Rollen der einzelnen Fertigkeiten neu bestimmt. Das Schreiben wird seitdem als eine komplexe Aktivität betrachtet, in der großer Wert auf die vielfältigen Aspekte des Schreibprozesses gelegt wird. Seit den 1980er Jahren wird dann das Schreiben mit steigendem Interesse erforscht (Mohr 2010: 991).

Der kommunikative bzw. postkommunikative Ansatz bringt in die sprachdidaktische Diskussion im Einklang mit der Entwicklung der Schreibdidaktik und sich etablierenden Modellen der Schreibentwicklung den Begriff Schreibkompetenz als Bezeichnung für die vollausgereifte Schreibfähigkeit (Boland 2005: 12) ein. Darunter versteht man die „Fähigkeit, sich anderen mitzuteilen und seine Gedanken schriftlich zu artikulieren und dabei zu entwickeln“" (Sieber 2003: 210). In Bezug auf das Fremdsprachenlernen und -lehren ist Schreibkompetenz ${ }^{1}$ als eine sprachenübergreifende Handlungskompetenz zu verstehen, die uns befähigt, Texte so zu verfassen, dass ihre Funktion und pragmatisch-kommunikative Absicht deutlich erkennbar wird (Sorger 2013b: 121).

In demselben Sinne ist der Begriff der Textkompetenz zu sehen. Aguado (2011: 107) bezeichnet Textkompetenz als Fähigkeit „Texte angemessen dekodieren und enkodieren zu können“. Sie weist darauf hin, „dass sprachliches Können meist modalitätsspezifisch unterschiedlich ausgebildetist", waswiederumheißt, dass manche Sprecher bei der Textrezeption oft erfolgreicher sind als bei der Textproduktion, bei anderen ist es dagegen umgekehrt. In der fremdsprachendidaktischen Diskussion haben sich dann zwei dichotome Begriffe etabliert und zwar rezeptive Textkompetenz und produktive Textkompetenz, die als wesentliche Grundlagen für eine erfolgreiche Textproduktion angesehen werden (vgl. Sorger 2013b: 121). Die Kompetenzen beziehen sich zwar auf die Erst- bzw. Muttersprache, man geht allerdings davon aus, dass sich diese Kompetenzen auch auf die nachfolgend gelernten Fremdsprachen übertragen.

1 Im selben Sinne verstehen wir die Schreibkompetenz in unserem Forschungsansatz, der in der Theorie des multiplen Sprachenlernens bzw. Sprachgebrauchs verortet ist. 


\section{Mehrsprachige Schreib- und Text(sorten)kompetenz in der L3-Forschung}

Aus der Sicht der Modelle zum multiplen Sprachenlernen (Hufeisen 2003) wird auch bei der Erforschung der mehrsprachigen Schreib- und Text(sorten)kompetenz davon ausgegangen, dass es in der L2 und L3 zu Transfer- und Interaktionsphänomenen zwischen den Interimssprachen (interlanguages) eines Individuums kommt. Genauso wird dabei in Betracht gezogen, dass bei der Entwicklung dieser Kompetenzen beim sukzessiven multiplen Fremdsprachenlernen die rekursiven und interaktiven Aspekte der Schreibprozesse mit steigender Kompetenz an Intensität zunehmen, wobei hier das deklarative sowie prozedurale Wissen aus mehreren Sprachen ständig interagiert (vgl. Sorger 2013b: 123). Entscheidend sind dabei in erster Linie folgende Faktoren, die einen Einfluss auf den Schreibprozess haben können:

- Chronologie des Sprachenerwerbs und das Lebensalter,

- produktiver Charakter des Schreibprozesses,

- die Stärke der Einprägung der beteiligten Sprachen,

- der Kontrast zur Erstsprache (Roche 2013: 160; Sorger 2013b: 124).

Die Schreib- und Text(sorten)kompetenz zählt in der Mehrsprachigkeitsbzw. Tertiärsprachenforschung zu den Bereichen, die in Bezug auf Sensibilisierungsmöglichkeiten untersucht werden müssten. Trotzdem fehlen hier weitestgehend Studien (vgl. auch Marx und Hufeisen 2010: 830), die die multiple Schreib- und Text(sorten)kompetenz und den zu erwartenden Transfer zwischen mehreren Sprachen untersuchen würden. Genau an diesem Punkt setzte unser Forschungsvorhaben an, das den Zusammenhang zwischen der Schreib- und Text(sorten)kompetenz von Schüler/-innen in der L1 und der Schreibkompetenz dieser Schüler/-innen in der L2 und L3 untersucht.

\section{Eine Untersuchung zur Ermittlung der mehrsprachigen Schreib- und Text(sorten)kompetenz bei tschechischen Schüler/-innen}

Auch in der Tschechischen Republik hat sich Englisch als erste Fremdsprache ${ }^{1}$ etabliert, wobei die anderen Fremdsprachen die Stellung von Folgefremdsprachen einnehmen. In den meisten Bildungssystemen, zu denen auch das tschechische gehört, wird Deutsch damit zur typischen zweiten Fremdsprache² (vgl. Sladkovská

1 Dies hängt damit zusammen, dass das Englische weltweit die Funktion einer lingua franca (d. h. „funktionales“ Verständigungsmittel) übernommen hat (Neuner 2003: 4).

2 Die Richtlinie der europäischen Sprachenpolitik von „Muttersprache plus zwei“ wird im tschechischen Bildungssystem erst seit dem Schuljahr 2013/14 wirklich umgesetzt, da erst seit diesem Zeitpunkt die zweite Fremdsprache verpflichtend ab der 8. Schulstufe angeboten werden muss (Sorger 2013a: 15). 
2010). Wie Pilypaityté (2013: 23) betont, ist diese Tatsache sowohl in der Fremdsprachenforschung als auch in der -didaktik nunmehr als allgemeiner Hintergrund anerkannt und die entsprechenden Schlussfolgerungen werden bereits gezogen. In Bezug auf die Unterrichtspraxis heißt dies, dass „eine intensive Unterstützung [notwendig wird], damit aus parallelen Unterrichtsfächern auch Synergien gezogen und das Lernen mehrerer Sprachen als ganzheitliches Konzept im Sinne der Tertiärsprachendidaktik angesehen wird" (Sorger 2013a: 15).

Die veränderte Stellung von $\mathrm{DaF}$ in der Tschechischen Republik gab den Anlass zu unserer empirischen Untersuchung zur Ermittlung der mehrsprachigen Schreib- und Textkompetenz bei tschechischen Schüler/-innen, die Tschechisch als L1, Englisch als L2 und Deutsch als L3 lernen. ${ }^{1}$ Fokussiert wurde hierbei auf die Entwicklung der soziolinguistischen und kommunikativen Kompetenz, wie sie in der modernen Fremdsprachendidaktik angestrebt und im Gemeinsamen Europäischen Referenzrahmen als ein wesentlicher Faktor der Sprachkompetenz angesehen wird. Die Analyse wurde auf der Basis geschriebener Texte durchgeführt und beleuchtet den in der Tertiärsprachenforschung bis dato kaum untersuchten Bereich der produktiven Text(sorten)kompetenz.

Im Zentrum des Interesses stand die Frage, ob es durch den Kontakt mit mehreren Sprachen einen produktiven Transfer ${ }^{2}$ im Bereich der textlinguistischen Kompetenz und der damit verbundenen Schreibkompetenz bei Lernenden gibt. Ein zentraler Aspekt der Untersuchung war die Ermittlung positiver Einflüsse aus der L1 auf die L2 und die L3 in der schriftlichen Produktion von tschechischen Lernenden. Um Textmaterial für die angestrebte Untersuchung zu gewinnen, wurden Schülern/-innen von zwei Grundschulen und zwei Gymnasien unterschiedliche Schreibaufgaben in den drei Sprachen Tschechisch, Englisch und Deutsch gestellt. So entstand ein Textkorpus, das 309 Texte jeweils in den drei Sprachen Tschechisch, Englisch und Deutsch zu insgesamt fünf Aufgabenstellungen umfasste (insgesamt 927 Texte). Es handelte sich dabei um fünf Textsorten, und zwar Notiz, Einladung E-Mail, Bildergeschichte und Beschreibung einer Handlung.

Die Auswertungskriterien sind der linguistische Textanalyse entlehnt. Für diese Analyse wurden solche sprachlichen Züge und Elemente registriert, die (1) typisch für die Beurteilung der Textproduktionskompetenz und (2) gemeinsam für alle hier untersuchten Textsorten bzw. auch Texttypen sind. Es wurden die Länge der Texte und makrostilistische sowie mikrostilistische Elemente registriert, die quantitativ und qualitativ ausgewertet wurden (mehr dazu Sorger 2013c: 86 ff.). Das

1 Diese Untersuchung wurde im Rahmen des Forschungsprojekts P407/11/0321 „Mnohojazyčnost $v$ české škole: učení a vyučování nèmčiny po angličtinè" (Mehrsprachigkeit in der tschechischen Schule: Deutschlernen und -lehren nach Englisch) durchgeführt.

2 Die Auseinandersetzung mit Transferprozessen beim multiplen Sprachenlernen hat zu einer umfassenden Ausdifferenzierung des Begriffs Transfer geführt. Meißner (2004:6) unterscheidet zwischen rezeptivem und produktivem Transfer, wobei sich diese Kategorienbildung aus der Vorgabe der jeweils betrachteten Fertigkeit ergibt. 
Forschungsverfahren und die Ergebnisse der im Rahmen des Forschungsprojektes durchgeführten Vergleichsstudien zu den einzelnen oben genannten Textsorten werden ausführlich in Sorger, Káňa, Janíková, Reitbrecht und Brychová (2013) vorgestellt.

Im Folgenden wird der Fokus auf eine der Voruntersuchungen zu dem im vorangegangenen Text kurz vorgestellten Forschungsvorhaben gelegt. Die Beschreibung dieser Voruntersuchung soll die Komplexität und den Facettenreichtum eines Forschungsvorhabens mit den genannten Zielen verdeutlichen.

\section{Voruntersuchungen zur Ermittlung der mehrsprachigen Schreib- und Text(sorten)kompetenz}

Im Folgenden werden ausgewählte Ergebnisse einer der Voruntersuchungen präsentiert, die sich auf die Textsorte schriftliche Einladung bezieht, und zwar im Hinblick darauf, wie diese Textsorte in curricularen Vorgaben, standardisierten Prüfungen, Sprachlehrwerken und im Sprachunterricht thematisiert wird. Die Datenerhebung schloss qualitative Methoden wie textlinguistische Analyse, Inhaltsanalyse, Interview mit den Lehrkräften sowie DaF-Studierenden und Aspekte der Auswertung ein. Das Hauptziel der Voruntersuchung war es, die Relevanz der schriftlichen Einladung als einer der funktional-kommunikativen Textsorten für die zentrale, textlinguistisch orientierte Untersuchung empirisch zu belegen (mehr dazu Janíková 2013: 139-166).

Bevor die Untersuchungsergebnisse zur Beantwortung der Frage, inwieweit der Texttyp Einladung in curricularen Vorgaben, standardisierten Prüfungen, Sprachlehrwerken und im Sprachunterricht thematisiert wird, vorgestellt werden, definieren wir diesen Texttyp kurz aus (fremd)sprachenunterrichtlicher und textlinguistischer Sicht. In Bezug auf den (Fremd)Sprachenunterricht betont Portmann-Tselikas (2010: 94), dass man ,viele Versuche, die Vielfalt der Texttypen und Schreibformen zu kategorisieren“ zur Verfügung hat. Aus textsortenstilistischer Sicht steht die Einladung dem Texttyp Brief sehr nahe. Sie zählt zu den handlungsbeeinflussenden und handlungspräformierenden Texten, die die appellative und handlungsvorbereitende Funktion erfüllen. Das heißt, die Verfasser/-innen appellierender Texte wollen bewusst und gezielt Einfluss auf ihre Adressaten nehmen, möchten zu Handlungen veranlassen oder diese verhindern und Reaktionen auslösen (sie haben eine starke perlokutive Kraft). Die Bandbreite appellativer (auffordernder) Texte reicht von einer Bitte, Annonce, Einladung, Handlungsanweisung bis hin zu Werbetexten.

Für die Einladung sind hinsichtlich der Makrostruktur folgende Merkmale charakteristisch: 
- Öffentlichkeitsgrad: Die Einladung ist für unterschiedliche Adressaten bzw. Adressatengruppen bestimmt und aus dieser Perspektive unterscheiden wir private und offizielle Einladungen.

- Kanal: Die Einladung kann entweder mündlich oder schriftlich formuliert werden.

- Ziel: Mit Texten, die als Einladung gelten, verfolgen die Verfasser/-innen zwei Hauptziele und zwar: Informieren und Veranlassen (Engel 1988: 47).

- Textgestaltung: Aus den Zielen einer schriftlichen Einladung lassen sich Forderungen an die Textgestaltung ableiten, die konventionalisiert ist und folgende Inhaltsschritte beinhaltet: Anrede, Thema, (Motivation), Abschiedsfloskeln, Unterschrift.

Schriftliche Einladungen werden in unterschiedlichen Formen gestaltet wie z. B. als Brief, E-Mail, Karte oder Plakat. Eine schriftliche Einladung, die in Briefform verfasst wird, veranlasst eine Person oder mehrere Personen zur Teilnahme an einer „Veranstaltung“. Sie enthält eine Orts- und Zeitangabe, den Anlass bzw. auch die entsprechende Motivation und erfordert von den Verfassern die Kenntnis geläufiger textsortenspezifischer Gestaltungsmerkmale. Eine Einladung erfüllt eine erkennbare kommunikative sowie interaktive Funktion, wobei sich besonders private Einladungen oft durch eine starke Emotionalität auszeichnen (Janíková 2013: 139).

Aus sprechakttheoretischer Sicht ist eine Einladung eine sprachliche Äußerung, die den direktiven Sprechakten zuzuordnen ist (vgl. Searle 1975). Durch die Äußerung versucht der Sprecher, den Adressaten zu einer bestimmten Handlung zu veranlassen. In der Passage, in der Searle die direktiven Sprechakte abgrenzt, wird die Einladung auch direkt genannt: „The illocutionary point of these [speech acts] consists in the fact that they are attempts [...] by the speaker to get the hearer to do something. They may be very modest, attempts' as when I invite you to do it or suggest that you do it, or they may be very fierce attempts as when I insist that you do it. [...] The propositional content is always that the hearer $\mathrm{H}$ does some future action A. Verbs denoting members of this class are ask, order, command, request, beg, plead, pray, entreat, and also invite, permit, and advise" (Searle 1975: 11; Herv. V. J.).

\subsection{Die Einladung in curricularen Vorgaben}

Für die Realisierung eines methodisch-didaktischen Ansatzes des muttersprachlichen sowie fremdsprachlichen Unterrichts sind curriculare Dokumente unterschiedlicher Art entscheidend. In ihnen erkennt man detailliertere Vorgaben für die Entwicklung der Schreib- und Textkompetenz, die häufig durch eine exemplarische Auflistung der zu behandelnden Textsorten bzw. -typen ergänzt sind. Uns hat interessiert, wie die Einladung in folgenden Dokumenten aufgegriffen wird: (1) in Profile Deutsch (2005), die die Referenzbeschreibung für das Erlernen und Lehren der deutschen 
Sprache konkretisieren, (2) im Rahmenbildungsprogramm für die Grundbildung (RVP ZV 2010) und (3) in den Bildungsstandards für die einzelnen Sprachen. Mittels einer Inhaltsanalyse sind wir zu folgenden Ergebnissen gelangt (vgl. Janíková 2013: 141-145):

ad 1) Profile Deutsch charakterisiert den Grad der Sprachbeherrschung und verweist darauf, wie gut Lernende auf einem bestimmten Niveau die gestellten Aufgaben lösen können. Die Kannbeschreibungen, die der Beschreibung von qualitativen Aspekten der sprachlichen Handlungsfähigkeit dienen, sind somit wichtige Orientierungshilfen bei der Zuordnung von Lernerproduktionen zu einem bestimmten Niveau und bei deren Beurteilung (Profile Deutsch, 2005: 102). Die Einladung wird zwar weder in den globalen noch in den detaillierten Kannbeschreibungen zur interaktiven schriftlichen Produktion auf dem Niveau A1 explizit genannt; trotzdem lassen sich die Charakteristika dieser Textsorte aus der globalen Kannbeschreibung eindeutig ablesen:

- A1: Globale Kannbeschreibungen - Interaktion schriftlich: „Kann kurze, einfache Texte schreiben, die zur Aufrechterhaltung von Sozialkontakten beitragen“ (Profile Deutsch 2005: 109).

Auf dem Niveau A2 enthalten die globalen Beschreibungen im Bereich der schriftlichen Interaktion Hinweise, die auf die Textsorte schriftliche Einladung passen:

- A2: Globale Kannbeschreibungen - Interaktion schriftlich: Kann kurze persönliche Texte, die Sozialkontakten dienen und sich in einfacher Form auf Wünsche beziehen, verstehen und mit einfachen sprachlichen Mitteln darauf reagieren (Profile Deutsch 2005: 118).

Darüber hinaus wird die „Einladung“ in den detaillierten Kannbeschreibungen explizit in den Beispielen genannt:

- A2: Detaillierte Kannbeschreibungen - Interaktion schriftlich: Kann sehr einfache persönliche Briefe, Postkarten und E-Mails schreiben und darin Persönliches austauschen.

Beispiel: Kann eine Freundin mit einer Postkarte zu ihrem Geburtstagsfest einladen (Profile Deutsch 2005: 119).

ad 2) Im tschechischen Rahmenbildungsprogramm für die Grundbildung (RVP ZV) wird die Einladung im Bildungsbereich "Sprache und sprachliche Kommunikation“ (Jazyk a jazyková komunikace) sowohl für Tschechisch im Muttersprachenunterricht 
als auch für den Fremdsprachenunterricht als verpflichtende Textsorte genannt (RVP ZV 2010).

ad 3) In der Tschechischen Republik wurden im Frühjahr 2013 die sog. Bildungsstandards für alle Fächer in der Grundbildung fertig gestellt und am 1. 9. 2013 der Lehrerschaft vorgelegt (Zařazení standardů do RVP ZV 2013). Sie sind als eine Erweiterung und Präzisierung der im Rahmenbildungsprogramm allgemein formulierten Anforderungen an die zu erreichenden Schulleistungen in den einzelnen Unterrichtsfächern zu verstehen. Für die Fremdsprachenlehrkräfte dienen sie als ein "Nachschlagewerk", denn sie beinhalten neben den ausführlichen Beschreibungen der zu erwartenden Ergebnisse auf der Niveaustufe A1 und A2 auch illustrative Aufgaben zur Entwicklung von Sprachkompetenz in Bezug auf die einzelnen Niveaustufen. In den Bildungsstandards für Deutsch, Englisch und Französisch als Fremdsprache (Niveaustufe A2, 9. Klasse, Sekundarstufe I) genauso wie in denjenigen für Tschechisch als Muttersprache kommt die Einladung als eine der zur Entwicklung der Schreibkompetenz empfohlenen Textsorten vor. Im Bildungsstandard für Tschechisch als Muttersprache kommt die Einladung in illustrativen Aufgaben schon für die 5. Klasse (letzter Jahrgang der Primarstufe) vor, hier aber nicht in schriftlicher, sondern in mündlicher Form.

\subsection{Die Einladung in standardisierten Prüfungen: tschechische Maturaprüfung in Fremdsprachen}

Die Analyse der tschechischen staatlichen Maturaprüfung in allen Fremdsprachen ergab, dass die Einladung zu den vorgeschriebenen Texttypen gehört. Es handelt sich um folgende Maturafächer: Deutsch, Französisch, Englisch, Italienisch, Russisch und Spanisch, die als Maturafächer bestimmt wurden (Nová maturita) (Janíková 2013: 141-142).

\subsection{Die Einladung in Lehrwerken und in der Unterrichtspraxis}

Bei der Lehrwerkanalyse hat uns interessiert, ob - und wenn ja - in welcher Form die Einladung in den Sprachlehrwerken für Tschechisch als Muttersprache (L1), Englisch als erste Fremdsprache (L2) und Deutsch als zweite Fremdsprache (L3) vorkommt. In den Interviews mit den an der Untersuchung beteiligten Sprachlehrkräften wurde die unterrichtspraktische Dimension einbezogen. Festgestellt wurde, ob und wann die Einladung im Unterricht thematisiert und geübt wird (Tabelle 1). 


\begin{tabular}{|c|c|c|c|c|}
\hline Klasse & Sprache & Lehrwerk & Einladung & $\begin{array}{l}\text { Training im Unterricht (im } \\
\text { Schuljahr, in dem die Untersuchung } \\
\text { durchgeführt wurde) }\end{array}$ \\
\hline \multirow[b]{4}{*}{$\begin{array}{l}\frac{0}{5} \\
\frac{1}{4} \\
\frac{1}{5} \\
\frac{1}{5} \\
n \\
N\end{array}$} & TCH-L1 & $\begin{array}{l}\text { Český jazyk } \\
\text { pro 7. ročník ZŠ }\end{array}$ & ja & $\begin{array}{l}\text { nein (Die schriftliche Einladung } \\
\text { wurde schon in den niedrigeren } \\
\text { Klassen im Zusammenhang mit } \\
\text { dem persönlichen Brief geübt; hier } \\
\text { nur mündliche Einladung.) }\end{array}$ \\
\hline & EN-L2 & Project new 2 & nein & $\begin{array}{l}\text { Die schriftliche Einladung wurde in } \\
\text { den niedrigeren Schulstufen geübt } \\
\text { (siehe auch Bildungsstandards). }\end{array}$ \\
\hline & \multirow{2}{*}{ DT-L3 } & $\begin{array}{l}\text { Deutsch mit } \\
\text { Max } 1\end{array}$ & $\begin{array}{l}\text { ja (schriftliche } \\
\text { Einladung zum } \\
\text { Geburtstag) }\end{array}$ & \multirow{2}{*}{ ja } \\
\hline & & Start mit Max 1 & $\begin{array}{l}\text { nein (aber als } \\
\text { strukturähnlicher } \\
\text { Texttyp "privater } \\
\text { Brief") }\end{array}$ & \\
\hline \multirow{4}{*}{ 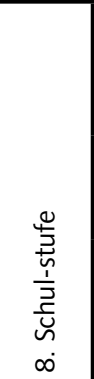 } & TCH-L1 & $\begin{array}{l}\text { Český jazyk } \\
\text { pro 8. ročník ZŠ }\end{array}$ & nein & $\begin{array}{l}\text { nein (Die schriftliche Einladung } \\
\text { wurde schon in den niedrigeren } \\
\text { Klassen im Zusammenhang mit } \\
\text { dem persönlichen Brief geübt.) }\end{array}$ \\
\hline & EN-L2 & Project 3 new & nein & nein \\
\hline & & Spaß mit Max 2 & nein & \multirow[b]{2}{*}{$\begin{array}{l}\text { nein (Die schriftliche Einladung } \\
\text { wurde in der } 7 . \text { Schulstufe geübt) }\end{array}$} \\
\hline & DT-L3 & $\begin{array}{l}\text { Deutsch mit } \\
\operatorname{Max} 1\end{array}$ & ja & \\
\hline
\end{tabular}

Tab. 1: Die Einladung in Sprachlehrwerken und im Sprachunterricht (Janíková 2013: 144)

Die Analyse der Lehrwerke zeigte, dass die Textsorte schriftliche Einladung in diesen Lehrwerken in unterschiedlichem Maße vorkommt. Im Lehrbuch für Tschechisch als L1 für die 7. Schulstufe wird die mündliche Einladung thematisiert, die schriftliche Einladung wurde nach Angaben der Lehrkräfte schon in den niedrigeren Klassen geübt, wobei diese Textsorte auch in den im Unterricht verwendeten Lehrbüchern erscheint. Sehr ähnlich ist die Situation in den Lehrbüchern für Englisch als L2 sowie im Englischunterricht, wo die „kurzen persönlichen Texte“ schon in niedrigeren Schulstufen intensiv geübt werden. In den im Deutsch als L3-Unterricht verwendeten Lehrbüchern ist die schriftliche Einladung präsent und wird in der 7. Jahrgangsstufe auch geübt. Dies bietet eine günstige Basis für die Aktivierung von Transferprozessen im Bereich der Textorganisation entlang des Vektors L1 $\rightarrow$ L2 $\rightarrow$ L3 (Janíková 2013: 145). Ob es in der Praxis zu solchen Transfererscheinungen kommt, muss noch weiter empirisch geprüft werden. 


\section{Zusammenfassung}

Als Zielfertigkeit hat das Schreiben im Fremdsprachenunterricht eine große Bedeutung, wurde aber in einzelnen methodischen Ansätzen unterschiedlich berücksichtigt. Eine ausgewogene Berücksichtigung aller Fertigkeiten bei der Entwicklung der fremdsprachlichen Kompetenz erscheint erst in der kommunikativen Sprachdidaktik, in noch höherem Maße manifestiert sich dies im postkommunikativen Ansatz, in dem funktional-kommunikativen Schreiben im Fremdsprachenunterricht eine besondere Bedeutung beigemessen wird. Eine neue Perspektive für das Schreiben eröffnet die Mehrsprachigkeitsdidaktik bzw. der Tertiärsprachendidaktik und zwar sowohl für den Fremdsprachenunterricht selbst als auch für seine Erforschung, in der aber leider bisher die Fertigkeit Schreiben bzw. die Schreibkompetenz noch zu wenig Raum einnimmt. Diese Tatsache bildete den Hintergrund für die in den Jahren 2012-2013 durchgeführte Untersuchung der mehrsprachigen Schreib- und Textkompetenz bei tschechischen Schüler/-innen, die Tschechisch als L1, Englisch als L2 und Deutsch als L3 lernen (siehe oben).

Um das Forschungsthema in seiner ganzen Komplexität aufgreifen zu können, wurden einige Voruntersuchungen durchgeführt, deren Ergebnisse als Teilaspekte der Auswertung in den einzelnen Studien zu fünf Textsorten (Einladung, Notiz, E-Mail, Handlungsbeschreibung und Erzählung) mitberücksichtigt wurden. In vorliegendem Artikel wurden die Ergebnisse einer vorbereitenden Untersuchung vorgestellt, deren Ziel es war festzustellen, wie und wann die schriftliche Einladung als einer der fünf in der Hauptstudie vertretenen Textsorten in curricularen Vorgaben, standardisierten Maturaprüfungen, Sprachlehrwerken sowie im Sprachunterricht aufgegriffen wird. Die Ergebnisse dieser Voruntersuchung zeigen, dass die schriftliche Einladung nach Angaben der Lehrkräfte in der Unterrichtspraxis trainiert wird. Sie wird außerdem in den analysierten Dokumenten sowie in den Unterrichts- und Prüfungsmaterialen oft explizit genannt. Dies bildet u. a. die sprachbildungsformale Voraussetzung dafür, dass der Produktionstransfer (Meißner 1998) beim Verfassen von Einladungstexten zumindest im konzeptionellen Rahmen der schulischen Fremdsprachenbildung gewährleistet werden kann (Janíková 2013: 144).

\section{Literaturverzeichnis}

Aguado, Karin (2011): Fremdsprachliche Textkompetenz - Zur Notwendigkeit der unterrichtlichen Förderung einer komplexen Fähigkeit. In: Bausch, Karl-Richard / Burwitz-Melzer, Eva / Königs, Frank G. (Hg.): Fremdsprachen lehren und lernen: Rückund Ausblick. Tübingen, Narr Verlag. S. 107-116.

Boland, Jan Hendrik (2005): Spezifische Schwierigkeiten des Deutschunterrichts an der Berufsschule bei der Vermittlung von Schreibkompetenz. Online: http://www.linse.unidue.de/linse/esel/pdf/boland_schreibkompetenz.pdf (22.6.2014).

Engel, Ulrich (1988): Deutsche Grammatik. Heidelberg, Julius Groos. 
Faistauer, Renate (2010): Die sprachlichen Fertigkeiten. In: Fandrych, Christian / Hufeisen, Britta / Krumm, Hans-Jürgen / Riemer, Clausia (Hg.): Deutsch als Fremdund Zweitsprache. Ein internationales Handbuch. (=Handbücher zur Sprach- und Kommunikationswissenschaft. Bd. 1). Berlin und New York, De Gruyter. S. 960-968.

Fandrych, Christian / Thurmaier, Maria (2011): Textsorten im Deutschen. Linguistische Analysen aus der sprachdidaktischen Sicht. Tübingen, Stauffenburg.

Funk, Hermann (2010): Methodische Konzepte für den Deutsch als FremdspracheUnterricht. In: Fandrych, Christian / Hufeisen, Britta / Krumm, Hans-Jürgen / Riemer, Clausia (Hg.): Deutsch als Fremd- und Zweitsprache. Ein internationales Handbuch. (=Handbücher zur Sprach- und Kommunikationswissenschaft. Bd. 1). Berlin und New York, De Gruyter. S. 939-951.

Hufeisen, Britta (2003): L1, L2, L3, L4, Lx - alle gleich? Linguistische, lernerinterne und lernerexterne Faktoren in Modellen zum multiplen Spracherwerb. In: Zeitschrift für Interkulturellen Fremdsprachenunterricht 8/2-3. S. 1-13. Online: http://zif.spz.tudarmstadt.de/jg-08-2-3/beitrag/Hufeisen1.htm (19.7.2014).

Janíková, Věra (2013): Studie I: Einladungsschreiben für einen Freund. In: Sorger, Brigitte / Káňa, Tomáš / Janíková, Věra / Reitbrecht, Sandra / Brychová, Alice (Hg.): Schreiben in mehreren Sprachen. Deutsch nach Englisch Mehrsprachigkeit und ihr Einfluss auf die Textkompetenz. Brno, Tribun. S. 139-166.

Kumaravadivelu, B. (2005): Understanding Language Teaching. From Method to Postmethod. New Jersey, Lawrence Erlbaum.

Marx, Nicole / Hufeisen, Britta (2010): Mehrsprachigkeitskonzepte. In: Fandrych, Christian / Hufeisen, Britta / Krumm, Hans-Jürgen / Riemer, Clausia (Hg.): Deutsch als Fremdund Zweitsprache. Ein internationales Handbuch. (=Handbücher zur Sprach- und Kommunikationswissenschaft. Bd. 1). Berlin und New York, De Gruyter. S. 825-831.

Meißner, Franz-Joseph (1998): Transfer beim Erwerb einer weiteren romanischen Fremdsprache: Das mehrsprachige mentale Lexikon. In: Meißner, Franz-Joseph / Reinfried, Marcus (Hg.): Mehrsprachigkeitsdidaktik. Konzepte und Erfahrungen mit der romanischen Mehrsprachigkeit im Unterricht (=Gießener Beiträge zur Fremdsprachendidaktik). Tübingen, Narr. S. 45-68.

Meißner, Franz-Joseph (2004): Transfer und Transferieren. Anleitungen zum Interkomprehensionsunterricht. Online: http://www.eurocomresearch.net/lit/Meissner Transfer.pdf (24. 6. 2014).

Mohr, Imke (2010): Vermittlung der Schreibfertigkeit. In: Fandrych, Christian / Hufeisen, Britta / Krumm, Hans-Jürgen / Riemer, Clausia (Hg.): Deutsch als Fremdund Zweitsprache. Ein internationales Handbuch. (=Handbücher zur Sprach- und Kommunikationswissenschaft. Bd. 1). Berlin und New York, De Gruyter. S. 991-997.

Neuner, Gerhard (2003): Mehrsprachigkeitskonzept (und Tertiärsprachendidaktik). Beispiel Englisch als erste und Deutsch als zweite Fremdsprache. In: per/voi, Januar bis Juni 2003. S. 4-11. Online: http://www.goethe.de/ins/it/pro/pervoi/pervoi12neu.pdf (22.5.2014).

Nová maturita. Online: http://www.novamaturita.cz/ (12. 1. 2014).

Portmann-Tselikas, Paul R. (2010): Schreiben. In: Hallet, Wolfgang / Königs, Frank G. (Hg.): Handbuch Fremdsprachendidaktik. Seelze-Veber, Kallmeyer/Klett. S. 92-96.

Profile Deutsch: Glaboniat, Manuela / Müller, Martin / Rusch, Paul / Schmitz, Helen / Wertenschlag, Lukas (2005): Profile Deutsch A1-C2. Gemeinsamer europäischer Referenzrahmen. Lernzielbestimmungen, Kannbeschreibungen, Kommunikative Mittel. Version 2.0. Berlin, Langenscheidt. 
Pilypaityte, Lina (2013): Schreiben in der Peripherie der Tertiärsprachenforschung und -didaktik. In: Sorger, Brigitte / Káňa, Tomáš / Janíková, Věra / Reitbrecht, Sandra / Brychová, Alice (Hg.): Schreiben in mehreren Sprachen. Deutsch nach Englisch Mehrsprachigkeit und ihr Einfluss auf die Textkompetenz. Brno, Tribun. S. 22-37.

Roche, Jörg (2013): Mehrsprachigkeitstheorie. Erwerb - Kognition - Transkulturation Ökologie. Tübingen, Narr Francke Attempto.

RVP ZV (2013): Rámcový vzdělávaci program pro základni vzděláváni. Online: http://www. nuv.cz/folder/18/display/ (29.6.2014).

Searle, John R. (1975). A classification of illocutionary acts. Language in Society 5/1, S. 1-23.

Sieber, Peter (2003): Modelle des Schreibprozesses. In: Bredel, Ursula / Günther, Hartmut / Klotz, Peter / Siebert-Ott, Gesa (Hg.): Didaktik der deutschen Sprache. Band 1. Paderborn, Schöningh (UTB). S. 208-223.

Sladkovská, Kamila (2010): Další cizí jazyk v českých školách. Online: http://clanky.rvp.cz/ clanek/c/JO/9557/DALSI-CIZI-JAZYK-V-CESKYCH-ZAKLADNICH-SKOLACH. $\mathrm{html} /(22.5 .2014)$.

Sorger, Brigitte (2013a): Fremdsprachen und Mehrsprachigkeit im tschechischen Schulunterricht. In: Sorger, Brigitte / Káňa, Tomáš / Janíková, Věra / Reitbrecht, Sandra / Brychová, Alice (Hg.): Schreiben in mehreren Sprachen. Deutsch nach Englisch Mehrsprachigkeit und ihr Einfluss auf die Textkompetenz. Brno, Tribun. S. 15-22.

Sorger, Brigitte (2013b): Schreibkompetenz und Textkompetenz. In: Sorger, Brigitte / Káňa, Tomáš / Janíková, Věra / Reitbrecht, Sandra / Brychová, Alice (Hg.): Schreiben in mehreren Sprachen. Deutsch nach Englisch Mehrsprachigkeit und ihr Einfluss auf die Textkompetenz. Brno, Tribun. S. 120-126.

Sorger, Brigitte (2013c): Forschungsdesign und Teilaspekte der Untersuchung. In: Sorger, Brigitte / Káňa, Tomáš / Janíková, Věra / Reitbrecht, Sandra / Brychová, Alice (Hg.): Schreiben in mehreren Sprachen. Deutsch nach Englisch Mehrsprachigkeit und ihr Einfluss auf die Textkompetenz. Brno, Tribun. S. 87-106.

Sorger, Brigitte / Káňa, Tomáš / Janíková, Věra / Reitbrecht, Sandra / Brychová, Alice (Hg.) (2013): Schreiben in mehreren Sprachen. Deutsch nach Englisch Mehrsprachigkeit und ihr Einfluss auf die Textkompetenz. Brno, Tribun. Zařazeni standardi do RVP ZV. 2013. Online: http: nuv.cz/ramcove-vzdelavaci-programy/zarazenistandardu-do-rvp-zv (1.6.2014).

\section{Lehrwerke}

Fišarová, Olga / Zbranková, Milena (2007): Deutsch mit Max 1. Plzeň, Fraus.

Fišarová, Olga / Zbranková, Milena (2000): Start mit Max 1. Plzeň, Fraus.

Hutchinson, Tom (2011a): Project new. Workbook 2. Oxford und New York, Oxford University Press.

Hutchinson, Tom (2011b): Project new. Pracovni sešit 2. Oxford und New York, Oxford University Press.

Hutchinson, Tom (2012a): Project new. Workbook 3. Oxford und New York, Oxford University Press.

Hutchinson, Tom (2012b): Project new. Pracovni sešit 3. Oxford und New York, Oxford University Press.

Tlustý, Petr / Cihlářová, Vítězslava / Wicke, Mariele, U. (2002): Spaß mit Max 2. Plzeň, Fraus. 
Styblík, Vlastimil / Čechová, Marie / Hauser, Přemysl / Hošnová, Eva (2000): Český jazyk pro 8. ročník základni školy a pro odpovidajici ročník viceletých gymnázii. Praha, SPN.

Styblík, Vlastimil / Čechová, Marie / Hauser, Přemysl / Hošnová, Eva (2001): Český jazyk pro 7. ročník základni školy a pro odpovidajici ročník víceletých gymnázii. Praha, SPN.

\author{
Věra Janíková \\ Katedra německého jazyka a literatur \\ Pedagogická fakulta MU \\ Poříčí 7 \\ 60300 Brno \\ janikova@ped.muni.cz
}

